\title{
Estudo do armazenamento da polpa do fruto ginja Eugênia uniflora L. e sua influencia nos teores de ácido ascórbico e antocianinas
}

\author{
Study of storage of fruit pulp of ginja Eugênia uniflora l. and their influences on levels of \\ ascorbic acid and anthocyanins
}

\author{
E. N. Modesto Junior ${ }^{1 *}$, S. S. Soares ${ }^{2}$, P. W. P. Gomes ${ }^{3}$, C. F. A. Ribeiro ${ }^{4}$, R. M. \\ V. da Silva ${ }^{5}$ \\ ${ }^{\text {I} P r o g r a m a ~ d e ~ P o ́ s-G r a d u a c ̧ a ̃ o ~ e m ~ C i e ̂ n c i a ~ e ~ T e c n o l o g i a ~ d e ~ A l i m e n t o s, ~ U n i v e r s i d a d e ~ F e d e r a l ~ d o ~ P a r a ́, ~ 66075-970 ~}$ \\ Belém-PA, Brasil. \\ ${ }^{2}$ Graduado em Tecnologia de Alimentos, Universidade do Estado do Pará, 68860-000.Salvaterra/Pará,Brasil \\ ${ }^{3}$ Programa de Pós-Graduação em Química, Universidade Federal do Pará, 66075-970 Belém-PA, Brasil. \\ ${ }^{4}$ Professora doutora em Engenharia Agrícola, Universidade do estado do Pará, 68860-000, Salvaterra/Pará, Brasil. \\ ${ }^{5}$ Mestre em Ciência e Tecnologia de Alimento, Técnico em Resíduos e Contaminantes, Laboratório Nacional \\ Agropecuário no Pará. 66093-02. Belém/Pará,Brasil. \\ *modesto.ufpa@outlook.com \\ (Recebido em 10 de abril de 2016; aceito em 28 de abril de 2016)
}

\begin{abstract}
A ginja (Eugenia uniflora L. - Myrtacea) é uma espécie vegetal endêmica da região Norte Fluminense e apresenta substâncias fenólicas com ação antioxidante, ação hipoglicemiante e antireumáticas, também são utilizadas em distúrbios estomacais e como anti-hipertensiva. O Brasil é o local onde a planta melhor se desenvolve, podendo atingir até oito metros de altura. O objetivo deste trabalho foi avaliar a melhor forma de armazenamento de polpa de ginja visando encontrar melhores condições para manutenção do teor de ácido ascórbico e antocianinas. Os frutos foram coletados no município de Salvaterra, Arquipélago do Marajó, Pará nas primeiras horas da manhã, sendo coletados manualmente das arvores. Em seguida, foram sanitizados a $10 \mathrm{ppm}$ e avaliados seus parâmetros físico-químicos e estudados quanto ao seu armazenamento. Para o armazenamento os frutos e polpa de ginja foram estudados por 60 dias em intervalos de $0,10,20,30$ e 60 dias sob temperatura de refrigeração $\left( \pm 8^{\circ} \mathrm{C}\right)$ e sob congelamento $\left( \pm-18{ }^{\circ} \mathrm{C}\right)$. Os frutos e as polpas armazenadas para cada tempo foram submetidos as análises de $\mathrm{pH}$, acidez titulável, umidade, cinzas, sólidos solúveis, ácido ascórbico e antocianinas totais. Em relação aos parâmetros físico-químicos estudados observou-se que sob refrigeração se teve uma maior variação dos resultados quando comparado ao congelamento tanto para o fruto quanto para a polpa de ginja. Concluiu-se que a melhor forma de armazenamento do fruto e da polpa de ginja foi sob congelamento, onde foi possível verificar que não houve perdas expressivas de ácido ascórbico e antocianinas.
\end{abstract}

Palavras-chave: Ácido ascórbico, Antocianinas, Armazenamento.

The sour cherry (Eugenia uniflora L. - Myrtacea) is a species endemic to the northern region of Rio de Janeiro and phenolic substances with antioxidant action presents, hypoglycaemic action and antireumatics are also used in stomach disorders and as antihypertensive. The Brazil it is the place where the best plant develops, reaching up to eight meters high. The aim of this work was to evaluate the best way of storage of apricot pulp in order to find better conditions for maintaining the content of Ascorbic acid and anthocyanins. The fruits were collected in the municipality of Salvaterra, Marajó Archipelago, Pará in the early hours of the morning, being collected manually from the trees. Then were sanitized to $10 \mathrm{ppm}$ and evaluated their physicochemical parameters and studied as to its storage. For storing fruit and apricot pulp were studied for 60 days at intervals of $0.10,20,30$ and 60 days under refrigeration temperature $\left( \pm 8{ }^{\circ} \mathrm{C}\right)$ and below freezing $\left( \pm-18{ }^{\circ} \mathrm{C}\right)$. The fruit and pulps stored for each time were submitted analyses of $\mathrm{pH}$, titulavel acidity, humidity, ashes, soluble solids, total anthocyanins and Ascorbic acid. In relation to the physical and chemical parameters studied showed that refrigerated if had a greater variation in the results when compared to freezing for both the fruit and the apricot pulp. It was concluded that the best way of storing the fruit and apricot pulp was under 
freezing, where it was possible to verify that there was no significant losses of Ascorbic acid and anthocyanins.

Keywords: Ascorbic acid, Anthocyanins, Storage.

\section{INTRODUÇÃO}

O consumo de frutas é associado à diminuição da incidência de câncer, diminuição da pressão arterial e de ocorrências cardiovasculares, sendo essa associação atribuída, principalmente, ao conteúdo em antioxidantes [1]. O Arquipélago do Marajó apresenta uma grande diversidade natural, destacando-se o potencial na produção de frutas das mais variadas características. Especificamente no município de Salvaterra, há vilas que produzem diversos frutos regionais. A maioria dos frutos estraga por falta de auxílio nas técnicas de armazenamento e falta de conhecimento das famílias produtoras. Algumas frutas e vegetais são considerados alimentos funcionais por apresentarem substâncias que trazem benefícios à saúde, como os compostos fenólicos (flavonoides, taninos, dentre outras) e a vitamina C (ácido ascórbico), os quais são considerados compostos antioxidantes [2,3]. Estas substâncias apresentam-se amplamente distribuídas no reino vegetal, por exemplo, o fruto de ginja.

A ginja (E. uniflora L.) é uma espécie vegetal endêmica da região Norte Fluminense [4] e apresenta substâncias fenólicas com ação antioxidante, hipoglicemiante e antireumáticas, também são utilizadas em distúrbios estomacais e como anti-hipertensiva [5]. Na região Norte o clima é muito propicio para o seu cultivo e no Marajó, especificamente em Salvaterra a produção de ginja é pouco disseminada, sendo encontrada principalmente nos quintais dos moradores locais e até mesmo em vias públicas.

Segundo Oliveira [5], o gênero Eugenia está entre os mais importantes da família Myrtaceae, com espécies de valor comercial, nutritivo e medicinal. No Nordeste brasileiro, sua importância econômica vem crescendo em virtude das suas excelentes propriedades sensoriais e elevados teores de vitamina A e C em frutos da família Myrtaceae (210 mg / 100 g e $14 \mathrm{mg} / 100 \mathrm{~g}$, respectivamente) [6]. Dessa forma o objetivo deste trabalho foi avaliar a melhor forma de armazenamento de frutos e de polpa de ginja visando encontrar melhores condições para manutenção do teor de ácido ascórbico e antocianinas.

\section{MATERIAL E MÉTODOS}

Os frutos de ginja foram obtidos no município de Salvaterra no Arquipélago do Marajó. Os mesmos foram coletados manualmente nas árvores para que não sofressem danos mecânicos, nas primeiras horas da manhã totalizando 100 frutos. Os frutos foram acondicionados em caixas plásticas para evitar injúrias no transporte, já que se trata de um fruto muito sensível.

Em seguida foram transportados até o Laboratório de Tecnologia de Alimentos da Universidade do Estado do Pará, Campus Salvaterra, onde passaram por um processo de seleção, separando os que apresentavam lesões dos sadios. Após esse processo os frutos foram sanitizados em água clorada a $10 \mathrm{ppm}$ por $10 \mathrm{~min}$ [7].

Para o estudo de armazenamento os frutos foram despolpados manualmente retirando-se a semente e mantendo a polpa aderida ao epicarpo por ser fino e estar bem aderida à polpa. Em seguida a polpa foi dividida em sacos de polietileno com aproximadamente $100 \mathrm{~g}$ cada e então submetidos à temperatura de refrigeração $\left( \pm 8^{\circ} \mathrm{C}\right)$ dias e congelamento $\left( \pm-18^{\circ} \mathrm{C}\right)$ por um período de 60 dias analisados em intervalos de $0,10,20,30,60$ dias.

Para a caracterização físico-química da polpa de ginja foram realizadas análises de $\mathrm{pH}$ [8], acidez titulável [8], umidade [9], cinzas [8], sólidos solúveis ( $\left.{ }^{\circ} B r i x\right) ~[8]$, ácido ascórbico [10] e antocianinas totais [11,12]. O tempo zero de armazenamento foi utilizado para comparação com os tratamentos. Em cada intervalo de estudo, a polpa foi retirada e analisada quanto aos parâmetros definidos em triplicata, de acordo com as metodologias sendo os resultados uma média de três repetições. 


\section{RESULTADOS E DISCUSSÃO}

Os resultados obtidos com os estudos de armazenamento para a polpa do fruto ginja ( $E$. uniflora $L$.) estão apresentados na Tabela 1.

Tabela 1: Estudo de armazenamento de polpa do fruto ginja (Eugênia uniflora L.) durante um período de dois meses sob refrigeração a $8{ }^{\circ} \mathrm{C}$.

\begin{tabular}{cccccc}
\hline Parâmetros & \multicolumn{5}{c}{ Tempo (Dias) } \\
\cline { 2 - 6 } pH & $\mathbf{0}$ & $\mathbf{1 0}$ & $\mathbf{2 0}$ & $\mathbf{3 0}$ & $\mathbf{6 0}$ \\
\cline { 2 - 6 } & $3,53^{\mathrm{a}} \pm 0,02$ & $3,25^{\mathrm{b}} \pm 0,02$ & $3,04^{\mathrm{d}} \pm \leq 1$ & $3,12^{\mathrm{c}} \pm 0,01$ & $3,12^{\mathrm{c}} \pm 0,04$ \\
Umidade (\%) & $85,88^{\mathrm{c}} \pm 0,62$ & $82,19^{\mathrm{d}} \pm 0,32$ & $89,64^{\mathrm{b}} \pm 0,14$ & $91,62^{\mathrm{a}} \pm 0,31$ & $90,70^{\mathrm{ab}} \pm 0,16$ \\
Acidez titulável & $0,044^{\mathrm{d}} \pm \leq 1$ & $0,189^{\mathrm{c}} \pm \leq 1$ & $0,40^{\mathrm{ab}} \pm \leq 1$ & $0,29^{\mathrm{b}} \pm 0,30$ & $0,43^{\mathrm{a}} \pm 0,44$ \\
$\quad(\%)$ & & & & & \\
Sólidos & $11,00^{\mathrm{a}} \pm \leq 1$ & $10,00^{\mathrm{b}} \pm \leq 1$ & $08,00^{\mathrm{c}} \pm \leq 1$ & $08,00^{\mathrm{c}} \pm \leq 1$ & $07,00^{\mathrm{c}} \pm 0,44$ \\
Solúveis ( ${ }^{\circ}$ Brix) & & & & & \\
Cinzas (\%) & $0,431^{\mathrm{a}} \pm \leq 1$ & $0,42^{\mathrm{a}} \pm 0,89$ & $0,26^{\mathrm{a}} \pm 0,30$ & $0,28^{\mathrm{a}} \pm 0,03$ & $0,39^{\mathrm{a}} \pm 0,08$ \\
Antocianinas & $179,27^{\mathrm{a}} \pm 1,80$ & $121,51^{\mathrm{b}} \pm 0,32$ & $9,52^{\mathrm{d}} \pm 0,21$ & $9,33^{\mathrm{d}} \pm 0,10$ & $59,38^{\mathrm{c}} \pm 0,04$ \\
(mg/100g) & & & & & \\
$\quad$ Ácido & $124,07^{\mathrm{a}} \pm 0,23$ & $79,83^{\mathrm{b}} \pm 1,67$ & $78,58^{\mathrm{b}} \pm 0,17$ & $28,95^{\mathrm{d}} \pm 0,05$ & $51,83^{\mathrm{c}} \pm 0,56$ \\
Ascórbico & & & & & \\
(mg/100g) & & & & & \\
\hline
\end{tabular}

Letras iguais na mesma linha não divergem significativamente no teste de Tuckey $(\mathrm{p}>0,05)$.

Pode-se observar na Tabela 1 que o $\mathrm{pH}$ apresentou decréscimo durante o armazenamento, interferindo para uma diferença significativa do tempo 0. Porém, os tempos 30 e 60 não diferem entre si e permaneceram constantes, dessa forma o fruto se apresenta com um caráter mais ácido com o passar do tempo de armazenamento. O teor de umidade se difere significativamente em função do tempo e pode-se perceber um aumento desse teor de 4,82\% duramente o armazenamento quando comparado o tempo 60 com o tempo 0 , porém os tempos 30 e 60 não apresentaram diferença significativa ao nível de $5 \%$. Verifica-se que durante o armazenamento somente os tempos 20 e 60 dias não diferem significativamente e é possível observar um acréscimo da acidez sob essas condições de armazenamento, Que pode estar relacionada à microbiota natural do fruto que interfere na sua estabilidade bioquímica. Durante o armazenamento dos frutos, os microorganismos fermentam os açúcares e os convertem em ácidos fermentando os açucares do fruto e os convertendo em ácidos orgânicos o que eleva o índice de acidez.

Com relação ao teor de sólidos solúveis do fruto de ginja houve diferença significativa entre os teores em função do tempo de armazenamento, com exceção apenas nos tempos 20 e 30 dias, Observa-se que no decorrer do tempo de armazenamento esse teor apresenta um decréscimo em comparação aos tempos 0 e 60 , isso se dá pelo fato de que os sólidos solúveis são os açucares do fruto que servem como substrato para os micro-organismos que os usam em seus processos fermentativos causando assim a queda observada duramente o armazenamento. Quanto ao teor de cinzas nos frutos de ginja em função do tempo de armazenamento não houve diferença significativa, embora se observe que houve uma perda desses minerais a partir do tempo de 20 dias de armazenamento sob refrigeração.

Com relação ao teor de antocianinas no fruto de ginja pode-se verificar que houve diferença significativa entre teor inicial e os demais tempos e pode ser observado que houve 
degradação desse pigmento durante o armazenamento e que após 2 meses houve uma redução intensa desse pigmento sobre refrigeração a $\pm 8{ }^{\circ} \mathrm{C}$. Vários fatores interferem na estabilidade de antocianinas (ACYS), incluindo $\mathrm{pH}$, ação de oxigênio, enzimas, variação de temperatura e incidência de luz [6]. A instabilidade térmica, a susceptibilidade desses compostos e a degradação são fatores que precisam ser investigados para garantir que suas propriedades e funcionalidades possam ser aproveitadas nas aplicações. Processos de degradação de ACYS podem levar a perda de cor e também a formação de compostos marrons insolúveis [13]. Estudos envolvendo a degradação de ACYS indicam a estabilidade de sucos de mirtilo (blueberry) a temperaturas de até $60{ }^{\circ} \mathrm{C}$ e destacam a aceleração da degradação com o aumento da temperatura. Amostras incubadas a $80,90,100,110$, e $115^{\circ} \mathrm{C}$ apresentaram perda de $50 \%$ das ACYS em intervalos de tempo de 180, 115, 40, 15 e 10 minutos [14]. Valores esses inferiores as perdas relatadas nesse estudo utilizando baixas temperaturas. O efeito do oxigênio na degradação das antocianinas foi constatado por Tressler e Pederson [15] citado por Kirca et al [16]. Esses autores observaram que a mudança da cor roxa para marrom em sucos de uva engarrafados podia ser prevenida simplesmente enchendo completamente as garrafas, ou seja, eliminando o oxigênio do seu interior. Verificou-se também na Tabela 1 que o tempo 60 apresentou um pico no teor de antocianinas o que contribui para sua diferença significativa e que pode ser explicado devido o estudo ter sido feito em lotes e que as ginjas não foram selecionadas quanto sua cor e a polpa não foi homogeneizada de forma adequada, o que pode ter ocasionado um erro de amostragem interferindo assim nesse resultado.

O teor inicial de ácido ascórbico no fruto de ginja variou de acordo com o tempo de armazenamento o que contribui para a diferença significativa entre os tempos comparados ao inicial. Não houve diferença significativa apenas nos tempos 20 e 30 dias. A sua degradação causando escurecimento, descoloração de pigmentos endógenos, perda ou mudança do sabor ou do odor, mudança na textura, e perda nutricional, está relacionada a diversos fatores como: oxigênio, $\mathrm{pH}$, luz, temperatura e conteúdo de umidade ou atividade de água e tem sido tema de diversos trabalhos $[17,18,19,20]$. O ácido ascórbico é conhecido por ser termolábil. Cinética de degradação térmica de sucos cítricos sob condições de pasteurização estabeleceram que ela segue um modelo de reação de $1^{\mathrm{a}}$ ordem [21]. Todas as frutas cítricas perdem vitamina $\mathrm{C}$ se armazenados a temperatura ambiente. A extensão da destruição de vitamina $\mathrm{C}$ dependerá do tipo de fruta. Albrecht et al [21] relataram que a retenção do AA (ácido ascórbico) variou de 56 a $98 \%$ para seis cultivares de brócolis armazenados a $2^{\circ} \mathrm{C}$ por 3 semanas. Retenção próxima as encontradas nesse estudo durante os dois meses de armazenamento que foi de $51,80 \%$ de ácido ascórbico comparado ao teor inicial.

$\mathrm{Na}$ Tabela 2 estão apresentados os resultados para o estudo de armazenamento da polpa congelada em um intervalo de dois meses. 
Tabela 2: Estudo de armazenamento de polpa do fruto ginja (Eugênia uniflora L.) durante um período de dois meses sob congelamento a $-18^{\circ} \mathrm{C}$.

\begin{tabular}{cccccc}
\hline \multirow{2}{*}{ Parâmetros } & \multicolumn{5}{c}{ Tempo (Dias) } \\
\cline { 2 - 6 } pH & $\mathbf{0}$ & $\mathbf{1 0}$ & $\mathbf{2 0}$ & $\mathbf{3 0}$ & $\mathbf{6 0}$ \\
\cline { 2 - 6 } & $3,53^{\mathrm{a}} \pm 0,02$ & $3,17^{\mathrm{ab}} \pm 0,01$ & $2,61^{\mathrm{b}} \pm 0,46$ & $3,27^{\mathrm{ab}} \pm 0,02$ & $2,94^{\mathrm{ab}} \pm 0,02$ \\
Umidade (\%) & $85,88^{\mathrm{c}} \pm 0,62$ & $81,77^{\mathrm{c}} \pm 0,64$ & $83,03^{\mathrm{c}} \pm 0,23$ & $81,74^{\mathrm{c}} \pm 0,17$ & $87,72^{\mathrm{a}} \pm 0,30$ \\
Acidez & $0,04^{\mathrm{d}} \pm \leq 1$ & $0,19^{\mathrm{b}} \pm 0,18$ & $0,19^{\mathrm{b}} \pm \leq 1$ & $0,21^{\mathrm{b}} \pm 0,01$ & $0,28^{\mathrm{a}} \pm 0,02$ \\
titulável (\%) & & & & & \\
Sólidos & $11,00^{\mathrm{a}} \pm \leq 1$ & $11,00^{\mathrm{a}} \pm \leq 1$ & $12,53^{\mathrm{a}} \pm 0,31$ & $11,80^{\mathrm{a}} \pm 1,77$ & $08,33^{\mathrm{c}} \pm 0,44$ \\
Solúveis & & & & & \\
('Brix) & & & & & \\
Cinzas (\%) & $0,43^{\mathrm{a}} \pm \leq 1$ & $0,20^{\mathrm{a}} \pm 0,06$ & $0,45^{\mathrm{a}} \pm 0,45$ & $0,50^{\mathrm{a}} \pm 0,45$ & $0,02^{\mathrm{a}} \pm 0,09$ \\
Antocianinas & $179,27^{\mathrm{a}} \pm 1,80$ & $171,90^{\mathrm{a}} \pm 0,89$ & $172,93^{\mathrm{a}} \pm 0,34$ & $97,87^{\mathrm{b}} \pm 0,45$ & $77,89^{\mathrm{c}} \pm 0,03$ \\
$($ mg/100g) & & & & & \\
Ácido & $124,07^{\mathrm{a}} \pm 0,23$ & $105,88^{\mathrm{a}} \pm \leq 1$ & $49,13^{\mathrm{c}} \pm 0,22$ & $111,46^{\mathrm{a}} \pm 0,25$ & $80,16^{\mathrm{b}} \pm 0,22$ \\
Ascórbico & & & & & \\
(mg/100g) & & & & & \\
\hline
\end{tabular}

Letras iguais na mesma linha não divergem significativamente no teste de Tuckey $(p>0,05)$.

Verifica-se na Tabela 2 que não houve diferença significativa entre o $\mathrm{pH}$ da ginja inicial tempo 0 e os tempos 10, 30 e 60 dias, diferindo dos demais apenas o tempo 20. Pode-se observar que não houve um decréscimo significativo no $\mathrm{pH}$ que neste caso foi quase mantido durante todos os meses de armazenamento.

Para o teor de umidade do fruto de ginja, não houve diferença significativa entre os tempos de 0 a 30 dias, havendo apenas diferença entre o tempo 60 dos demais. Verificou-se também que esse teor se manteve estável durante um mês de congelamento tendo um aumento apenas no último mês de estudo.

Com relação a acidez do fruto de ginja sob congelamento houve diferença significativa entre tempo 0 e demais tempos, entretanto, não houve diferenças entre os tempos 10, 20 e 30. Verifica-se que houve um acréscimo da acidez durante o armazenamento tendo um maior pico no tempo 60 com uma variação de $0,04 \%$ a $0,28 \%$.

Observa-se na Tabela 2 que para os teores de sólidos solúveis na polpa de ginja congelada não houve diferenças significativas entre os tempos 0 a 30 dias de armazenamento, porém houve diferença entre o tempo 60 dos demais. Pode-se observar que teor de açucares do fruto se manteve estável durante um mês de armazenamento decaindo somente no segundo mês de estudo. O conteúdo de cinzas não apresentou diferença significativa entre os tempos durante os meses de armazenamento.

Para os teores de antocianinas na polpa de ginja congelada verificou-se que não houve diferença significativa entre os tempos de 0 a 20 dias, diferindo apenas os tempos de 30 e 60 dias entre si. Pode-se verificar que durante o tempo de armazenamento da polpa de ginja o teor na quantidade de pigmentos se manteve estável, decrescendo somente no final do armazenamento. Nesse processo pode-se avaliar uma perda de $28,54 \%$ do teor de pigmentos durante os dois meses de estudo comparados ao teor inicial. Os valores determinados no tempo zero foram superiores aos encontrados por Lima et al [22] em polpas de diferentes genótipos de aceroleira, $(6,4$ a $64,6 \mathrm{mg} / 100 \mathrm{~g})$.

Segundo Cheftel et al [23], durante o congelamento reações metabólicas são reduzidas, porém não totalmente inativadas, pode-se dessa forma então justificar degradação dos 
pigmentos antociânicos em polpas armazenadas por esse processo. Agostini-Costa et al [24] embora o objetivo principal do estudo tenha sido avaliar o efeito do processo industrial de congelamento de polpa de acerola e avaliar a estabilidade dos carotenoides durante a estocagem, estes autores perceberam que o monitoramento químico do teor de antocianinas acusou uma perda de $14 \%$ após um ano de congelamento, valor abaixo do encontrado nesse estudo. Avaliando a estabilidade do teor de antocianinas totais na polpa de frutos de 6 clones de aceroleira, armazenada sob congelamento, relataram que houve redução no teor desses pigmentos de até $36,2 \%$ [25].

Verifica-se na Tabela 2 que houve diferença significativa entre os teores de ácido ascórbico encontrados na polpa de ginja nos tempos 20 e 60 dias em relação aos demais tempos estudados. $\mathrm{O}$ decréscimo do tempo 20 pode ser explicado devido o estudo ter sido feito em lotes e esse lote possivelmente se apresentava desde o início do armazenamento com alguma deficiência no teor de ácido ascórbico que quando influenciado pelos fatores de armazenamento contribui para perda significativa nesse teor. Após 4 meses de armazenagem, as acerolas armazenadas a $-12{ }^{\circ} \mathrm{C} \mathrm{e}-18{ }^{\circ} \mathrm{C}$ apresentaram teores de $869 \pm 12$ e $1.223 \pm 148 \mathrm{mg}$ vit.C/100 g, respectivamente, representando uma perda de $43 \%$ e $19 \%$, respectivamente, em relação ao teor inicial [26]. Verificou-se no presente estudo que durante o armazenamento sob temperaturas de congelamento pode-se verificar uma perda de apenas 30,16\% do teor inicial de AA, se mostrando um bom método para conservação desse composto por períodos não tão longos, sendo valores próximos ao do estudo de Yamashita et al [26].

Silva et al. [27], perceberam que o armazenamento da polpa de cagaita, congelada em um intervalo de estudo de quatro meses, nas condições testadas, apresentou decréscimo no teor de vitamina C, com uma redução de $33,7 \%$, valores próximos ao encontrados nesse trabalho para ginja. No Brasil, estimasse uma ingestão diária recomendada (IDR) de vitamina $\mathrm{C}$ para adultos de $60 \mathrm{mg}$ [28]. Dessa forma a polpa congelada durante os dois meses de armazenamento conseguiu manter valores acima do valor diário recomendado.

Comparando-se os resultados das Tabelas 1 e 2 em relação aos parâmetros físicoquímicos estudados pode-se perceber que os obtidos para refrigeração variaram mais que para os obtidos no estudo de congelamento do fruto indicando ser o melhor método para conservação das características naturais dos frutos de ginja nos aspectos físico-químicos e nutricionais. Os compostos fitoquímicos são sensíveis à temperatura logo o teor de antocianinas e ácido ascórbico podem ter sido influenciados pela temperatura de refrigeração e congelamento como pode ser observado nas Figuras 1 e 2.

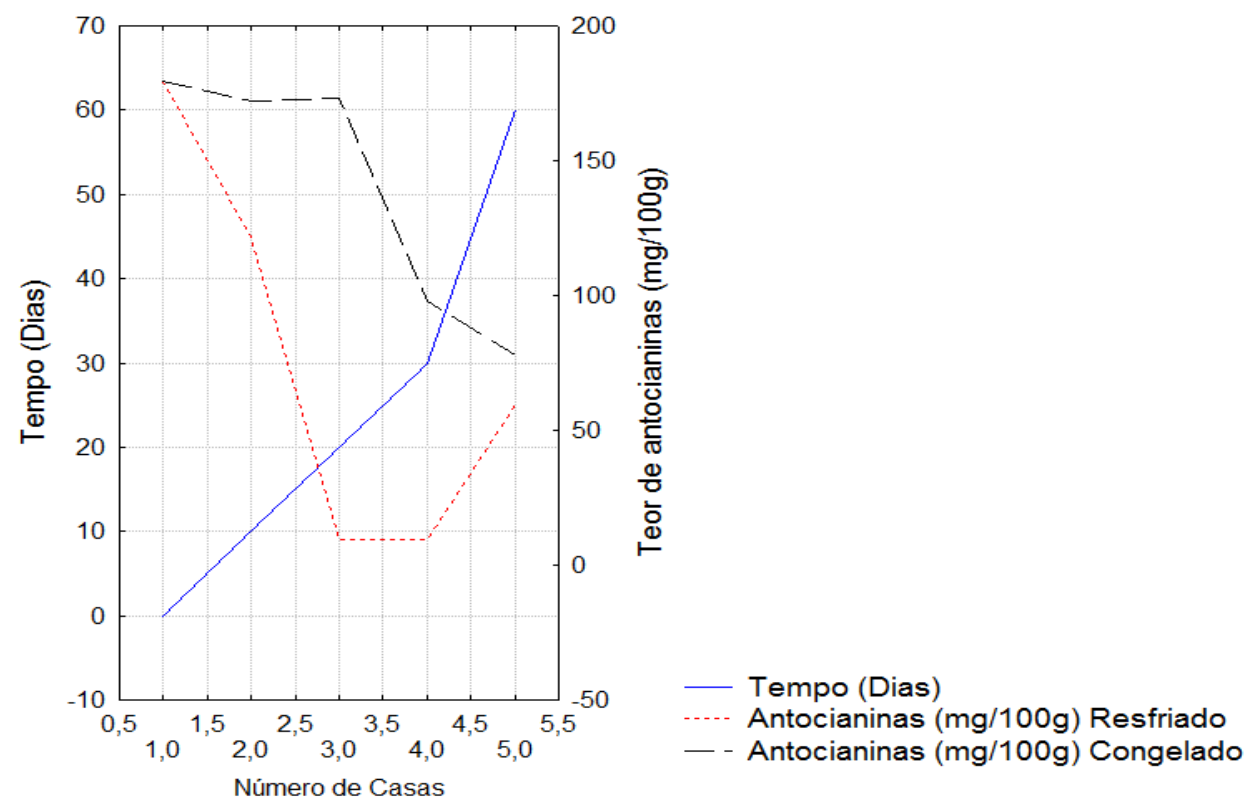

Figura 1: Influência da refrigeração e congelamento no armazenamento de ginja sob os teores de antocianinas. 


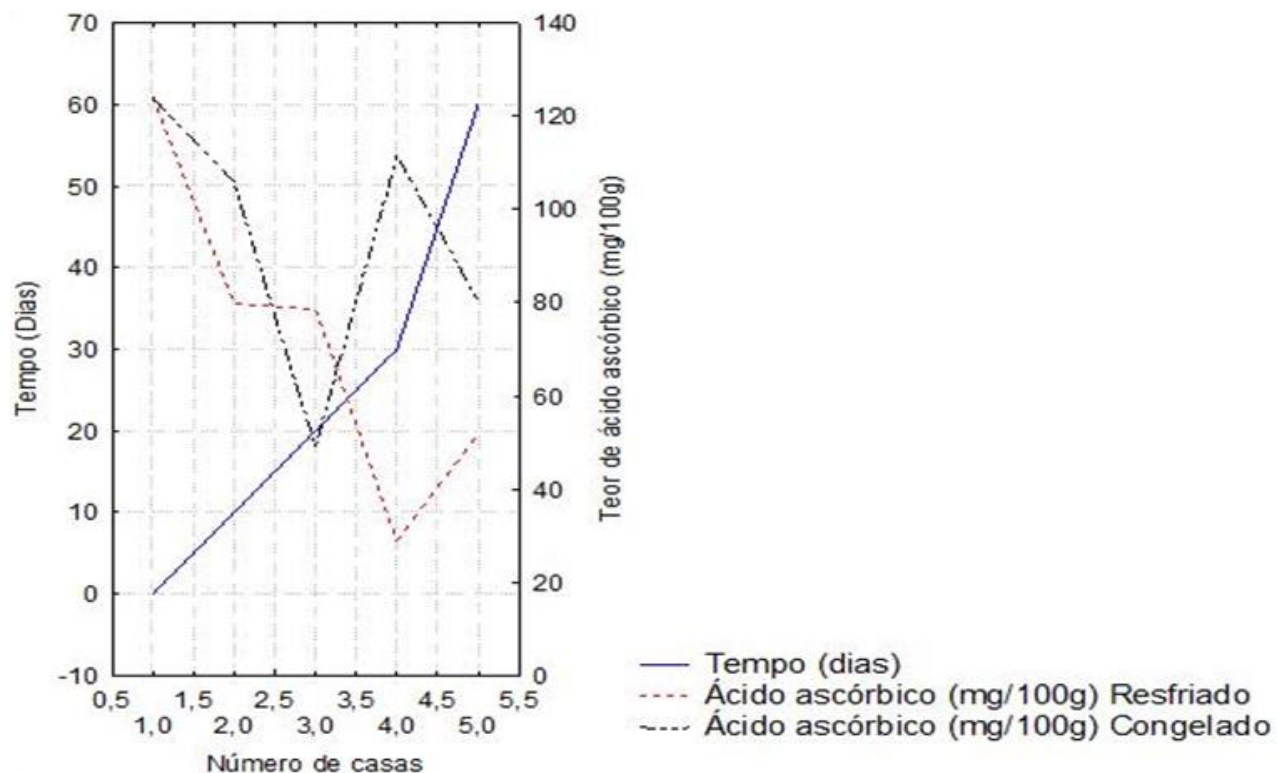

Figura 2: Influência da refrigeração e congelamento no armazenamento de ginja sob os teores de ácido ascórbico.

As antocianinas sob refrigeração apresentaram $72,47 \%$ de perda dos pigmentos onde foi também possível perceber visivelmente a descoloração do vermelho para tons de marrom após 10 dias sob refrigeração. Já sob congelamento os resultados foram mais satisfatórios pois se obteve uma perda de $20,68 \%$ dos pigmentos de antocianinas, valor inferior a perda constatada sob refrigeração. Os teores de AA sob refrigeração apresentaram um rápido decréscimo, dessa forma sobre refrigeração se teve $51,80 \%$ de perda de AA comparado ao teor inicial e em temperaturas negativas foi possível perceber uma perda de apenas $33 \%$ do teor de AA do teor inicial, mantendo resultados acimas do que a legislação exige como valores ingestão diária de $60 \mathrm{mg} / \mathrm{dia}$.

\section{CONCLUSÃO}

No estudo do armazenamento da polpa de ginja menores perdas de ácido ascórbico, antocianinas, melhores características sensórias e físico-químicas foram encontradas no armazenamento do fruto congelado. Logo para se manter por mais tempo a estabilidade dos compostos fotoquímicos presentes na polpa a melhor alternativa e mantê-los em temperaturas igual ou mais baixa que $-18^{\circ} \mathrm{C}$.

\section{REFERÊNCIAS BIBLIOGRÁFICAS}

1. Inoque T, Komoda H, Uchida T, Node K. Tropical fruit camu-camu (Myrciaria dúbia) has antioxidant and anti-inflammatory properties. Journal of Cardiology. 2008; 52(1): 127-132. doi:10.1016/j.jjcc.2008.06.004.

2. Broinizi PRB, Andrade-Wartha, ERS, Silva AMO, Novoa AJV, Torres RP, Azeredo HMC, Alves RE, Mancini-Filho J. Avaliação da atividade antioxidante dos compostos fenólicos naturalmente presentes em subprodutos do pseudofruto de caju (Anacardium occidentale L.). Ciência e Tecnologia de Alimentos. 2007; 27(4): 890-896.

3. Ajaikumar KB, Asheef M, Babu BH, Padikkala J. The inhibition of gastric mucosal injury by Punica granatum L. (pomegranate) methanolic extract. J. Ethnopharmacol. 2005; 96:171-76.

4. Assumpção J, Nascimento MT. Estrutura e composição florística de quatro formações vegetais de restinga no complexo lagunar Grussaí/Iquipari, São João da Barra, RJ, Brasil. Acta Botânica Brasil. 2005; 14: 301-315. doi:10.1016/j.jep.2004.09.007. 
5. Oliveira FMN, Figuêiredo RMF, Queiroz AJM. Análise comparativa de polpas de pitanga integral, formulada e em pó. Revista Brasileira de Produtos Agroindustriais. Campina Grande. 2006; 8(1): 25-33.

6. Alighourchi H, Barzegar M. Some physicochemical characteristics and degradation kinetic of anthocyanin of reconstituted pomegranate juice during storage. Journal of Food Engineering. 2009; 90 (2): 179-185. doi: 10.1016/j.jfoodeng.2008.06.019

7. Andrade NJ, Macêdo JAB. Higienização na indústria de alimentos. São Paulo: Varela. 1996. 182p.

8. Instituto Adolfo Lutz. Normas Analíticas do Instituto Adolfo Lutz: Métodos químicos para análises de alimentos. São Paulo, 2005.

9. Association of Official Analytical Chemists. Official methods of analysis of the Association of Official Analytical Chemists (method 920.39,C). Arlington: A.O.A.C., 1995, chapter 33. p. $10-12$.

10. Silva SLA, Ferreira GAL, Silva RR. À procura da vitamina C. Química Nova na Escola. 1(2): 31 32, 1995.

11. Askar A, Treptow H. Quality assurance in tropical fruit processing. New York: Springer-Verlag, 1993. 231p.

12. Rogez H. Açaí: Preparo composição e melhoramento da conservação. Belém: Editora da Universidade Federal do Pará - EDUFPA, 2000. 313p.

13. Wang WD, Xu SY. Degradation kinetics of anthocyanins in blackberry juice and concentrate. Journal of Food Engineering. 2007; 82(1): 271-275. doi:10.1016/j.jfoodeng.2007.01.018.

14. Buckow R, Kastell A, Terefe NS, Versteeg C. Pressure and Temperature Effects on Degradation Kinetics and Storage Stability of Total Anthocyanins in Blueberry Juice. Journal of Agricultural and Food Chemistry. 2010; 58(1): 10076-10084. doi: 10.1021/jf1015347.

15. Kirca A, Özkan M, Cemeroglu B. Stability of black carrot anthocyanins in various fruit juices and nectars. Food Chemistry. 2006; 101(1): 212-218. doi:10.1016/j.foodchem.2005.05.036.

16. Yamashita F, Benassi MT, Kieckbusch TG. Effect of modified atmosphere packaging on kinetics of vitamin C degradation in mangos. Brazilian Journal Food Technology. 1999; 1(18): 127-130.

17. Prado MET, Chandra PK, Bicalho UO. Desenvolvimento de um modelo matemático para estimar a degradação da vitamina $\mathrm{C}$ durante armazenamento de alimentos de umidade intermediaria. Ciência e Tecnologia de Alimentos. 1995; 159(1): 138-143.

18. Zanoni B, Peri C, Nani R, Lavelli V. Oxidative heat damage of tomato halves as affected by drying. Food Research International. 1999; 31(1): 395-401. doi:10.1016/S0963-9969(98)00102-1.

19. Carvalho AV, Lima LCO. Qualidade de kiwis minimamente processados e submetidos a tratamento com ácido ascórbico, ácido cítrico e cloreto de cálcio. Pesquisa Agropecuária Brasileira. 2002.; 37(5): 679-685.

20. Riguetto AM. Caracterização físico-química e estabilidade do suco de acerola verde microencapsulado por atomização e liofilização. [Dissertação] Faculdade de Engenharia de Alimentos, Universidade Estadual de Campinas - UNICAMP, 176 p. 2003.

21. Albrecht JA, Schafer HW, Zottola EA. Sulfhydryl and ascorbic acid relationships in selected vegetables and fruits. Journal of Food Scientific. 1991; 1(56): 427-430. doi: 10.1111/j.13652621.1991.tb05296.x

22. Lima VLAG, Mélo EA, Guerra NB. Correlação entre o teor de antocianinas e caracterização cromática de polpas de diferentes genótipos de aceroleira. Brazilian Journal Food of Technology. 2007; 10(1): 51-55.

23. Cheftel JC, Cheftel H, Besançon P. Métodos de conservacíon. In: Cheftel, J. C.; Cheftel, H.; Besançon, P. (Ed.) Introduccion a la bioquímica y tecnología de los alimentos. Zaragoza: Acribia. 1983; 2(1): 173-202.

24. Agostini-Costa TS, Abreu LN, Rossetti AG. Efeito do congelamento e do tempo de estocagem da polpa de acerola sobre o teor de carotenóides. Revista Brasileira de Fruticultura. 2003; 25(1): 5658. doi: S0100-29452003000100017.

25. Araújo PGL, Figueiredo RW, Alves RE, Maia GA, Paiva JR. $\beta$. - caroteno, ácido ascórbico e antocianinas totais em polpa de frutos de aceroleira conservada por congelamento durante 12 meses. Ciência e Tecnologia de Alimentos. 2007; 27(1): 104-107.

26. Yamashita F, Benassi MT, Tonzar AC, Moriya S, Fernandes JG. Produtos de acerola: estudo da estabilidade de Vitamina C. Ciência e Tecnologia de Alimentos. 2003; 23(1): 92-94.

27. Silva FCC, Viana AP, Silva MGO. Caracterização química e Determinação dos estádios fenológicos de variedades de videiras cultivadas no Norte Fluminense. Revista Brasileira de Fruticultura [online]. 2008; 30(1): 38-42.

28. Brasil. Portaria SVS/MS n 33, de 13 de janeiro de 1998. Tabelas de Ingestão Diária Recomendada (IDR). Diário Oficial da União de 16 de janeiro de 1998. 